\title{
Young's Modulus of Single-Crystal Fullerene $C_{70}$ Nanotubes
}

\author{
Tokushi Kizuka, ${ }^{1}$ Kun'ichi Miyazawa, ${ }^{2}$ and Takayuki Tokumine ${ }^{1}$ \\ ${ }^{1}$ Institute of Materials Science, Graduate School of Pure and Applied Sciences, University of Tsukuba, \\ Tsukuba 305-8753, Japan \\ ${ }^{2}$ Fullerene Engineering Group, Materials Processing Unit, National Institute for Materials Science, Namiki, \\ Tsukuba 305-0044, Japan \\ Correspondence should be addressed to Tokushi Kizuka, kizuka@ims.tsukuba.ac.jp
}

Received 1 June 2011; Accepted 22 July 2011

Academic Editor: Ilia A. Solov'yov

Copyright (c) 2012 Tokushi Kizuka et al. This is an open access article distributed under the Creative Commons Attribution License, which permits unrestricted use, distribution, and reproduction in any medium, provided the original work is properly cited.

\begin{abstract}
We performed bending tests on single-crystal nanotubes composed of fullerene $\mathrm{C}_{70}$ molecules by in situ transmission electron microscopy with measurements of loading forces by an optical deflection method. The nanotubes with the outer diameters of 270-470 nm were bent using simple-beam and cantilever-beam loading by the piezomanipulation of silicon nanotips. Young's modulus of the nanotubes increased from $61 \mathrm{GPa}$ to $110 \mathrm{GPa}$ as the outer diameter decreased from $470 \mathrm{~nm}$ to $270 \mathrm{~nm}$. Young's modulus was estimated to be $66 \%$ of that of single-crystal $\mathrm{C}_{60}$ nanotubes of the same outer diameter.
\end{abstract}

\section{Introduction}

There have been many investigations of crystal morphologies and structures of fullerene $\mathrm{C}_{60}$ molecules since the first report on the crystallization of fullerene $\mathrm{C}_{60}$ molecules. $\mathrm{C}_{60}$ crystals have been shown in the forms of plates, films, and rods [1-13]. In particular, single-crystal fullerene nanowhiskers (NWs) with high length-to-diameter aspect ratios have been synthesized by a liquid-liquid interfacial precipitation (LLIP) method $[14,15]$. In addition, tubular fullerene NWs, that is, single-crystal fullerene nanotubes (NTs) have been synthesized by the same method [16]. The mechanical properties of NWs and NTs have been investigated for application to structural materials and functional devices. Buckling tests of $\mathrm{C}_{60}$ NWs and NTs have been performed by in situ transmission electron microscopy (TEM), and their Young's moduli were estimated to be 53-69 GPa and 62$107 \mathrm{GPa}$, respectively [17-19]. Fullerene NWs and NTs can be synthesized using another well-known fullerene molecule, that is, $C_{70}[15,20]$. The mechanical nature of fullerene NWs and NTs can be controlled by selecting their constituent molecules, as demonstrated in fullerene films [21, 22]. In this study, we performed bending tests on $\mathrm{C}_{70}$ NTs by in situ TEM.

\section{Experimental}

We synthesized $\mathrm{C}_{70}$ NTs by the LLIP method using a saturated solution of $\mathrm{C}_{70}$ molecules in pyridine and 2-propanol [16]. The solution, including precipitated $\mathrm{C}_{70} \mathrm{NTs}$, was added dropwise to the edge of a gold plate. The plate was mounted on a specimen holder of the transmission electron microscope equipped with a piezomanipulation system at the University of Tsukuba [23-26]. A silicon microcantilever with a nanometer-sized tip used for contact-type atomic force microscopy (AFM) was fixed onto a cantilever holder. Both the specimen and the cantilever holders were inserted into the microscope. The cantilever tip was brought into contact with individual NTs fixed on the plate edges by piezomanipulation inside the microscope. The tip was then pressed on the NTs for bending with simple-beam- and cantilever-beam-type loading. The cantilever tip was then pulled back to release the force. A series of these manipulations were performed several times at room temperature in a vacuum of $1 \times 10^{-5} \mathrm{~Pa}$ using the same NT. The deformation process was observed in situ using a television and video capture system with a time resolution of $17 \mathrm{~ms}$. At the same time, the force applied to the NTs was measured by optical detection of the cantilever deflection used in AFM. 
The spring constant of the cantilever was measured to be $4.7 \mathrm{~N} / \mathrm{m}$.

\section{Results}

Figure 1 shows a time-sequence series of the bright-field images of the bending process of a $\mathrm{C}_{70} \mathrm{NT}$ protruding from an edge of the gold plate. The dark triangular region in the upper part of each frame of Figure 1 is the cantilever tip. The brighter region around the NT is the vacuum. The outer and inner diameters of the NT in Figure 1 are $270 \mathrm{~nm}$ and $100 \mathrm{~nm}$, respectively. The length of the deformed part is $2.5 \mu \mathrm{m}$. The crystal structure of the NT was tetragonal. This crystal structure has been observed in dried $\mathrm{C}_{70} \mathrm{NWs}$, $\mathrm{C}_{60}$ NWs, and NTs synthesized by LLIP methods [18-20]. Polymerized $\mathrm{C}_{70}$ crystals under hydrostatic compression have an orthorhombic structure with similar lattice constants $a$ and $c$, which is comparable to the tetragonal structure [27]. The longer growth axis of the NT is aligned parallel to the [110] direction. The left side of the NT was fixed on the gold plate, as shown in Figure 1. In this bending test, the cantilever tip was initially placed in contact with the NT (Figure 1(a)). Then, force was exerted on the NT in the direction indicated by the arrow in Figure 1(a) and was bent (Figure 1(b)). Thus, bending of the NT was performed using cantilever-beamtype loading. Subsequently, the tip was released, and the NT recovered its initial straight shape (Figure 1(c)). This observation shows that the bending is an elastic deformation. This bending and recovery cycle was repeated several times using the same NT. Figure 2 shows the relationship between the force and the flexure during the bending process depicted in Figure 1. The points indicated by arrowheads $\mathrm{a}-\mathrm{c}$ in Figure 2 correspond to the TEM images in Figures $1(\mathrm{a})-1(\mathrm{c})$. The maximum flexure and loading at the free end of the NT were $467 \mathrm{~nm}$ and $431 \mathrm{nN}$, respectively. A hysteresis is observed in Figure 2 during the pressing and pulling of the cantilever tip. This is attributed to the bonding of the cantilever tip with the NT surface [28]. Sawtooth-like variations are observed in both the pressing and pulling processes. The decrease in force during the pressing process and the increase in force during the pulling process are caused by the rotation of the cantilever tip due to slip of the contact region between the cantilever tip and the NT surface [28]. The increase in force during the pulling process arises from the recovery of deflection of the AFM cantilever beam by increase in the recovery shift of the NT due to slip. The other changes in the exerted force, that is, the increase during the pressing process and the decrease during the pulling process, are not significantly influenced by slip events. On the basis of the relationships among force, flexure, and Young's modulus in cantilever-beam loading, we estimated that Young's modulus of the NTs is 68-110 GPa from the slope of the force curve in these regions. We performed a bending test with the same type loading for another NT with an outer diameter of $470 \mathrm{~nm}$ and an inner diameter of $230 \mathrm{~nm}$. Its Young's modulus was estimated to be $61 \pm 5 \mathrm{GPa}$.

Figure 3 shows a time-sequence series of the bright-field images of the bending process of a $\mathrm{C}_{70} \mathrm{NT}$, both ends of

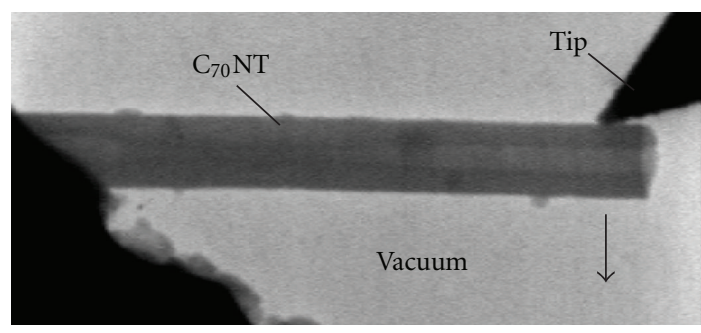

(a)

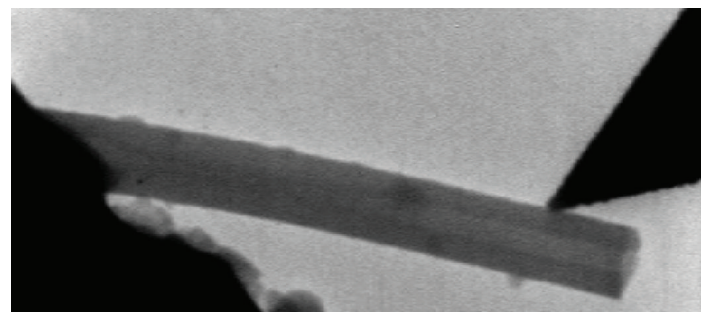

(b)

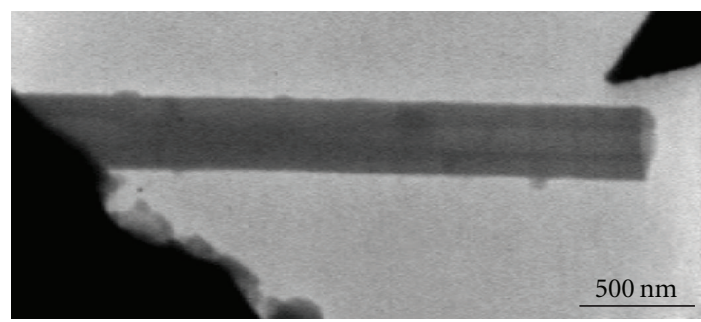

(c)

FIGURE 1: A time-sequence series image and the separation like galley is forbidden. Time-sequence series of bright-field images of cantilever-beam-type loading bending of single-crystal $\mathrm{C}_{70}$ nanotube with outer diameter of $270 \mathrm{~nm}$ and inner diameter of $100 \mathrm{~nm}$. One end of the nanotube is fixed on a gold plate. The arrow indicat es the direction of loading imparted by the tip of AFM cantilever. This bending test was performed in the vacuum.

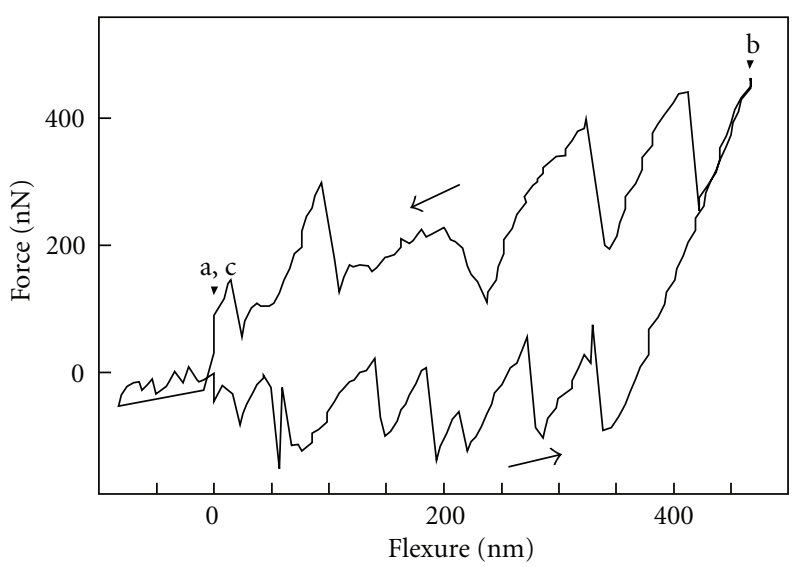

Figure 2: Force-flexure curve of $\mathrm{C}_{70}$ nanotube during bending shown in Figure 1. Points a-c correspond to the states shown in Figures $1(\mathrm{a})-1(\mathrm{c})$. The arrows indicate the time path of recording. 


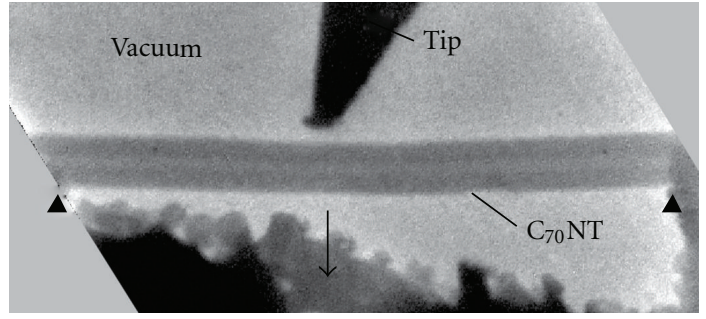

(a)

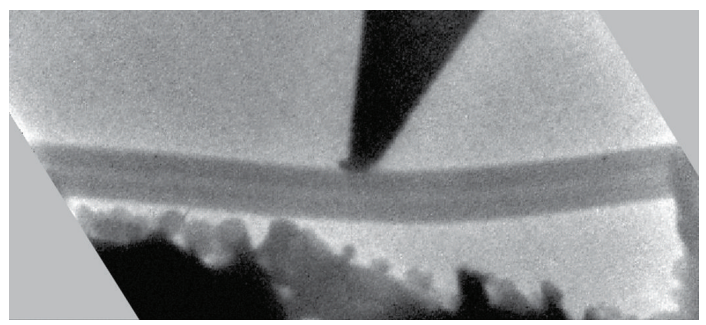

(b)

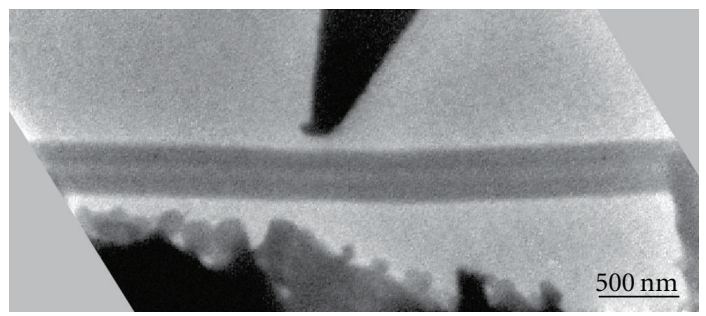

(c)

Figure 3: Time-sequence series of bright-field images of loading of simple-beam-type bending of single-crystal $\mathrm{C}_{70}$ nanotube with outer diameter of $340 \mathrm{~nm}$ and inner diameter of $80 \mathrm{~nm}$. The nanotube is supported on a gold plate with two fulcra indicated by the triangles. The arrow indicates the loading direction by the tip of the AFM cantilever. This bending test was performed in the vacuum.

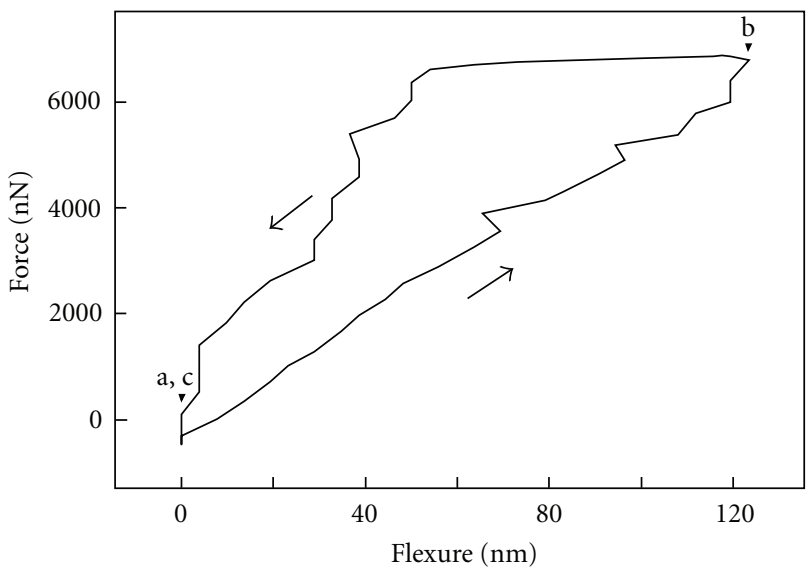

Figure 4: Force-flexure curve of $\mathrm{C}_{70}$ nanotube during bending shown in Figure 3. Points a-c correspond to the states shown in Figures $3(\mathrm{a})-3(\mathrm{c})$, respectively. The arrows indicate the time path of recording.

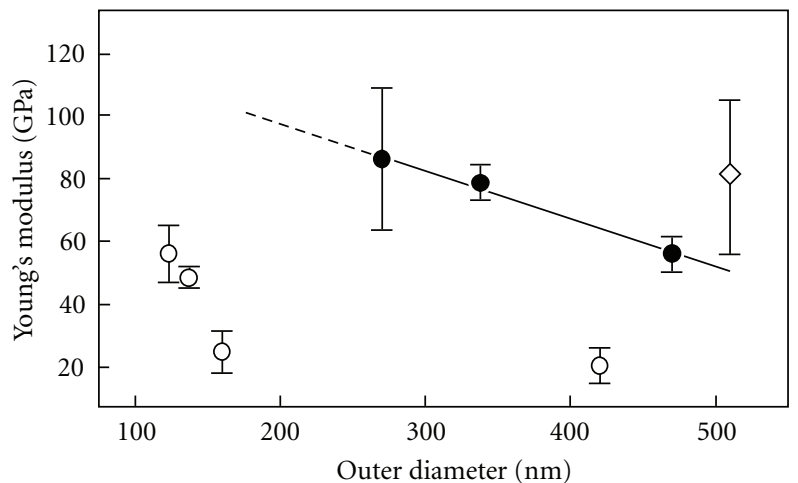

Figure 5: Young's modulus of single-crystal $C_{70}$ nanotubes plotted against outer diameter. The values observed in the present study are indicated by the filled circles. The solid line indicates a linear approximation. For reference, Young's moduli of single-crystal $C_{60}$ nanotubes and nanowhiskers are represented with rhombus and open circles, respectively $[18,19,29]$.

which were fixed on the gold plate. The outer and inner diameters of the NT are $340 \mathrm{~nm}$ and $80 \mathrm{~nm}$, respectively. The length of the region of deformation was $3.5 \mu \mathrm{m}$. The loading was performed at the center of the deformation region by the cantilever tip. Thus, this loading was simple-beam-type loading. Figure 4 shows the relationship between the force and the flexure during the bending process presented in Figure 2. The maximum flexure and loading at the free end were $123 \mathrm{~nm}$ and $6.8 \mu \mathrm{N}$, respectively. Similar to the cantileverbeam-type bending in Figure 1, a hysteresis is observed. The pressing process from state (a) to (b) in Figure 4 is approximated by one slope. In contrast, the pulling process from state (b) to (c) in Figure 4 is composed of two slopes. The lower slope from state (b) in the pulling process is attributed to the friction between the cantilever tip and the NT surface. This is because the rotation of the cantilever tip, that is, the change in cantilever-beam deflection, is suppressed by the friction [28]. From the slope during the pressing process from state (a) to (b) in Figure 4, we estimated Young's modulus of the NTs to be $82 \pm 5 \mathrm{GPa}$ on the basis of the relationships among force, flexure, and Young's modulus in simple-beam loading.

In Figure 5, we plotted Young's modulus of these $\mathrm{C}_{70}$ NTs against their outer diameters. Young's modulus increases as the outer diameter decreases. The relationship between Young's modulus $E(\mathrm{GPa})$ of $\mathrm{C}_{70}$ NTs and the outer diameters $d(\mathrm{~nm})$ is approximated by a straight line, $E=-0.143 d+129$, as shown in Figure 5. As the thickness of the NTs used in this study is $85-130 \mathrm{~nm}$, the tubular structure does not occur when the minimum outer diameter decreases less than $170-260 \mathrm{~nm}$. From this minimum diameter, the maximum Young's modulus of $\mathrm{C}_{70}$ NTs is estimated to be $92-105 \mathrm{GPa}$. Kizuka et al. reported that Young's modulus of a $\mathrm{C}_{60}$ NT with an outer diameter of $510 \mathrm{~nm}$ is $84.5 \mathrm{GPa}$ [18]. From the linear approximation in Figure 5, Young's modulus of a $\mathrm{C}_{70}$ NT with the same outer diameter, that is, $510 \mathrm{~nm}$, is calculated to be $56 \mathrm{GPa}$. Thus, Young's modulus of the $\mathrm{C}_{70} \mathrm{NT}$ is $66 \%$ of that of a $\mathrm{C}_{60} \mathrm{NT}$ of the same outer diameter. 


\section{Discussion}

4.1. Young's Modulus of $C_{70}$ NTs and Films. Young's moduli of $\mathrm{C}_{70}$ NTs estimated in this study are 7-28 times larger than those of crystalline $\mathrm{C}_{70}$ films (4-9 GPa) $[21,22]$. The crystal lattice of the $\mathrm{C}_{70}$ NTs studied here is tetragonal, whereas crystal structure of $\mathrm{C}_{70}$ crystalline films is the face-centered-cubic structure $[21,22]$. The averaged center-to-center distance between adjoining $\mathrm{C}_{70}$ molecules in the NTs along the growth direction is $3 \%$ smaller than that of $\mathrm{C}_{70}$ NTs with a face-centered-cubic structure [15]. According to Miyazawa et al., this difference can be attributed to the polymerization of the molecules [14]. Thus, it is deduced that the increase in Young's modulus observed in this study originates from the effect of the polymerization of the constituent $\mathrm{C}_{70}$ molecules, as previously discussed for the bending tests of $\mathrm{C}_{60} \mathrm{NTs}$ [18].

4.2. Young's Modulus of $C_{70}$ NTs and $C_{60}$ NTs. The estimated Young's moduli of $\mathrm{C}_{70}$ NTs are larger than those of the crystalline $\mathrm{C}_{70}$ films, whereas the modulus obtained for the $\mathrm{C}_{70}$ NT with an outer diameter of $510 \mathrm{~nm}$ is $66 \%$ of that of a $\mathrm{C}_{60}$ NT with the same diameter. Kolomenskii et al. and Murugavel et al. reported a similar quantitative relationship of Young's moduli in crystalline $\mathrm{C}_{70}$ and $\mathrm{C}_{60}$ films $[21,22]$. They attribute this relationship to the difference in molecular densities of crystalline $\mathrm{C}_{70}$ and $\mathrm{C}_{60}$ films.

4.3. Dependency of Young's Modulus on the Outer Diameter. As shown in Figure 5, Young's modulus increases as the outer diameter decreases. Saito et al. observed a similar relationship between Young's modulus and the outer diameter of $\mathrm{C}_{60}$ NWs [19]. They discussed the relationship on the basis of a core-shell structure model of $\mathrm{C}_{60}$ NWs. Ringor and Miyazawa investigated the incubation process of $\mathrm{C}_{60} \mathrm{NWs}$ in solution and revealed that $\mathrm{C}_{60}$ NTs were formed owing to the elution of the interior regions [30]. Kato and Miyazawa pointed out from the cross-sectional TEM that the density of pores in the interior region of $\mathrm{C}_{60} \mathrm{NWs}$ is higher than that in the region near the surfaces [31]. Their result also sup-ports the core-shell structure of NWs. The dependency of the Young's modulus on the outer diameter, as shown in Figure 5, infers that the core component remained in the $\mathrm{C}_{70}$ NTs and the larger Young's modulus of thinner NTs is attributed to a smaller amount of the core component, which leads to a lower density of the NT.

\section{Conclusion}

We performed bending tests on individual $\mathrm{C}_{70}$ NTs by in situ TEM. From the measurements of the force-flexure relationships of $\mathrm{C}_{70}$ NTs, Young's modulus was estimated to be 61$110 \mathrm{GPa}$. Young's modulus increased as the outer diameter decreased. From this relationship, the Young's modulus of a $\mathrm{C}_{70}$ NT was estimated to be $66 \%$ of that of a $\mathrm{C}_{60}$ NT of the same outer diameter, that is, $510 \mathrm{~nm}$. The maximum Young's modulus of $\mathrm{C}_{70}$ NTs was estimated to be $92-105 \mathrm{GPa}$ from the limit of the thickness of the tubular structure.

\section{Acknowledgment}

This study was partly supported by Grants-in-Aid from the Ministry of Education, Culture, Sport, Science, and Technology, Japan (nos. 22310065 and 23651127).

\section{References}

[1] W. Krätschmer, L. D. Lamb, K. Fostiropoulos, and D. R. Huffman, "Solid $\mathrm{C}_{60}$ : a new form of carbon," Nature, vol. 347, no. 6291, pp. 354-358, 1990.

[2] W. I. F. David, R. M. Ibberson, J. C. Matthewman et al., "Crystal structure and bonding of ordered $\mathrm{C}_{60}$," Nature, vol. 353, no. 6340, pp. 147-149, 1991.

[3] S. J. Duclos, K. Brister, R. C. Haddon, A. R. Kortan, and F. A. Thiel, "Effects of pressure and stress on $\mathrm{C}_{60}$ fullerite to 20 GPa," Nature, vol. 351, no. 6325, pp. 380-382, 1991.

[4] P. A. Heiney, J. E. Fischer, A. R. McGhie et al., "Orientational ordering transition in solid $\mathrm{C}_{60}$," Physical Review Letters, vol. 66, no. 22, pp. 2911-2914, 1991.

[5] R. L. Meng, D. Ramirez, X. Jiang et al., "Growth of large, defect-free pure $\mathrm{C}_{60}$ single crystals," Applied Physics Letters, vol. 59, no. 26, pp. 3402-3403, 1991.

[6] W. Krakow, N. M. Rivera, R. A. Roy, R. S. Ruoff, and J. J. Cuomo, "Epitaxial growth of $\mathrm{C}_{60}$ thin films on mica," Journal of Materials Research, vol. 7, no. 4, pp. 784-787, 1992.

[7] X. D. Shi, A. R. Kortan, J. M. Williams, A. M. Kini, B. M. Savall, and P. M. Chaikin, "Sound velocity and attenuation in singlecrystal $\mathrm{C}_{60}$," Physical Review Letters, vol. 68, no. 6, pp. 827-830, 1992.

[8] Y. Yosida, "Scanning electron microscope images of $\mathrm{C}_{60}$ whiskers," Japanese Journal of Applied Physics, vol. 31, no. 4B, pp. L505-L507, 1992.

[9] M. Haluška, H. Kuzmany, M. Vybornov, P. Rogl, and P. Fejdi, "A double-temperature-gradient technique for the growth of single-crystal fullerites from the vapor phase," Applied Physics A, vol. 56, no. 3, pp. 161-167, 1993.

[10] J. Z. Liu, J. W. Dykes, M. D. Lan, P. Klavins, R. N. Shelton, and M. M. Olmstead, "Vapor transport growth of $\mathrm{C}_{60}$ crystals," Applied Physics Letters, vol. 62, no. 5, pp. 531-532, 1993.

[11] J. L. D. Boer, S. V. Smaalen, V. Petricek, M. DusekP, M. A. Verheijen, and G. Meijer, "Hexagonal close-packed $\mathrm{C}_{60}$," Chemical Physics Letters, vol. 219, no. 5-6, pp. 469-472, 1994.

[12] F. Michaud, M. Barrio, S. Toscani et al., "Solid-state studies on single and decagonal crystals of $\mathrm{C}_{60}$ grown from 1,2-dichloroethane," Physical Review B, vol. 57, no. 17, pp. 10351-10358, 1998.

[13] S. Toscani, H. Allouchi, J. L. Tamarit et al., "Decagonal $\mathrm{C}_{60}$ crystals grown from n-hexane solutions: solid-state and aging studies," Chemical Physics Letters, vol. 330, no. 5-6, pp. 491496, 2000.

[14] K. Miyazawa, Y. Kuwasaki, A. Obayashi, and M. Kuwabara, " $\mathrm{C}_{60}$ nanowhiskers formed by the liquid-liquid interfacial precipitation method," Journal of Materials Research, vol. 17, no. 1, pp. 83-88, 2002.

[15] K. Miyazawa, K. Hamamoto, S. Nagata, and T. Suga, "Structural investigation of the $\mathrm{C}_{60} / \mathrm{C}_{70}$ whiskers fabricated by forming liquid-liquid interfaces of toluene with dissolved $\mathrm{C}_{60} / \mathrm{C}_{70}$ and isopropyl alcohol," Journal of Materials Research, vol. 18, no. 5, pp. 1096-1103, 2003. 
[16] J. Minato and K. Miyazawa, "Solvated structure of $\mathrm{C}_{60}$ nanowhiskers,” Carbon, vol. 43, no. 14, pp. 2837-2841, 2005.

[17] K. Asaka, R. Kato, K. Miyazawa, and T. Kizuka, "Buckling of $\mathrm{C}_{60}$ whiskers," Applied Physics Letters, vol. 89, no. 7, Article ID 071912, 3 pages, 2006.

[18] T. Kizuka, K. Saito, and K. Miyazawa, "Young's modulus of crystalline $\mathrm{C}_{60}$ nanotubes studied by in situ transmission electron microscopy," Diamond and Related Materials, vol. 17, no. 6, pp. 972-974, 2008.

[19] K. Saito, K. Miyazawa, and T. Kizuka, "Bending process and Young's modulus of fullerene $\mathrm{C}_{60}$ nanowhiskers," Japanese Journal of Applied Physics, vol. 48, no. 1, Article ID 010217, 3 pages, 2009.

[20] K. Miyazawa, " $\mathrm{C}_{70}$ nanowhiskers fabricated by forming liquid/liquid interfaces in the systems of toluene solution of $\mathrm{C}_{70}$ and isopropy/alcohol," Journal of the American Ceramic Society, vol. 85, no. 5, pp. 1297-1299, 2002.

[21] A. A. Kolomenskii, M. Szabadi, and P. Hess, "Laser diagnostics of $\mathrm{C}_{60}$ and $\mathrm{C}_{70}$ films by broadband surface acoustic wave spectroscopy," Applied Surface Science, vol. 86, no. 1-4, pp. 591596, 1995.

[22] P. Murugavel, C. Narayana, A. Govindaraj, A. K. Sood, and C. N. R. Rao, "Brillouin scattering from $\mathrm{C}_{70}$ and $\mathrm{C}_{60}$ films: a comparative study of elastic properties," Chemical Physics Letters, vol. 331, no. 2-4, pp. 149-153, 2000.

[23] T. Kizuka, K. Yamada, S. Deguchi, M. Naruse, and N. Tanaka, "Cross-sectional time-resolved high-resolution transmission electron microscopy of atomic-scale contact and noncontacttype scannings on gold surfaces," Physical Review B, vol. 55, no. 12, pp. R7398-R7401, 1997.

[24] T. Kizuka, "Atomic process of point contact in gold studied by time-resolved high-resolution transmission electron microscopy," Physical Review Letters, vol. 81, no. 20, pp. 44484451, 1998.

[25] T. Kizuka, "Direct atomistic observation of deformation in multiwalled carbon nanotubes," Physical Review B, vol. 59, no. 7, pp. 4646-4649, 1999.

[26] T. Kizuka, "Atomic configuration and mechanical and electrical properties of stable gold wires of single-atom width," Physical Review B, vol. 77, no. 15, Article ID 155401, 11 pages, 2008.

[27] A. V. Soldatov, G. Roth, A. Dzyabchenko et al., "Topochemical polymerization of $\mathrm{C}_{70}$ controlled by monomer crystal packing," Science, vol. 293, no. 5530, pp. 680-683, 2001.

[28] S. Fujisawa and T. Kizuka, "Effect of lateral displacement of atomic force microscope tip caused by contact scanning studied by in situ transmission electron microscopy," Japanese Journal of Applied Physics, vol. 42, no. 3, pp. L1182-L1184, 2003.

[29] K. Asaka and T. Kizuka, "Atomistic dynamics of deformation, fracture, and joining of individual single-walled carbon nanotubes," Physical Review B, vol. 72, no. 11, Article ID 115431, 5 pages, 2005.

[30] C. L. Ringor and K. Miyazawa, "Fabrication of solution grown $\mathrm{C}_{60}$ fullerene nanotubes with tunable diameter," Journal of Nanoscience and Nanotechnology, vol. 9, no. 11, pp. 6560-6564, 2009.

[31] R. Kato and K. Miyazawa, "Cross-sectional structural analysis of $\mathrm{C}_{60}$ nanowhiskers by transmission electron microscopy," Diamond and Related Materials, vol. 20, no. 3, pp. 299-303, 2011. 

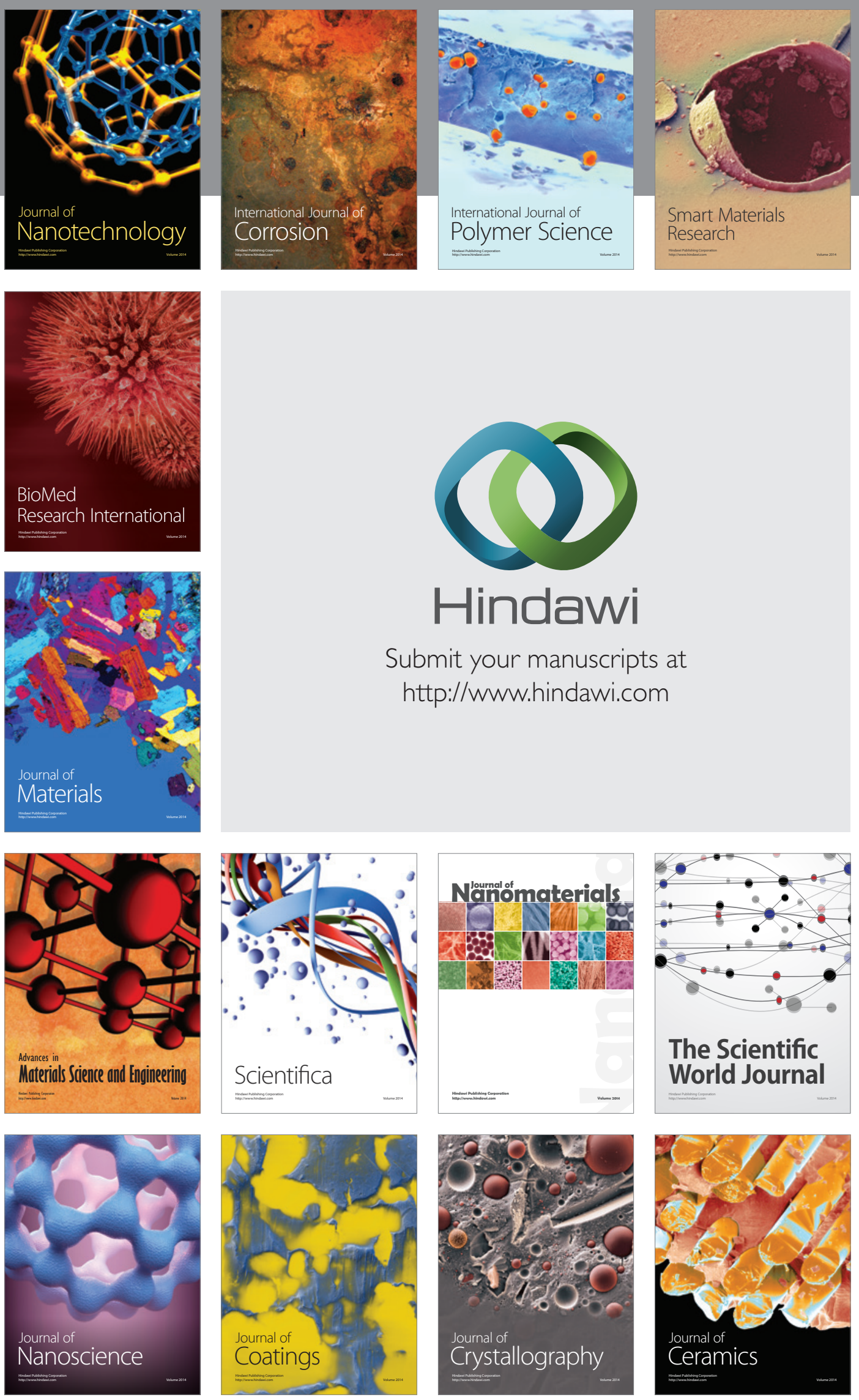

The Scientific World Journal

Submit your manuscripts at

http://www.hindawi.com

\section{World Journal}

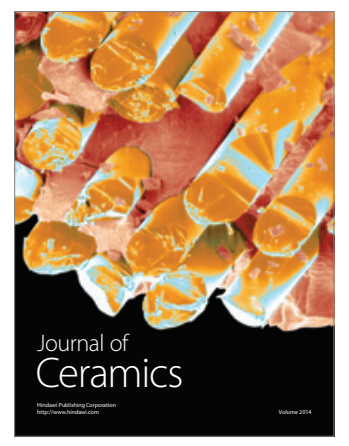

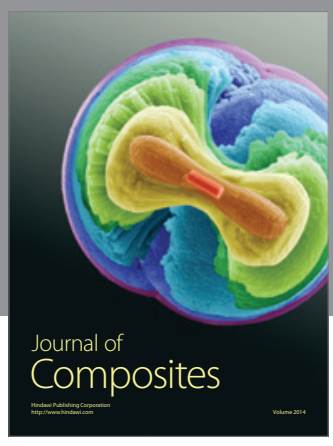
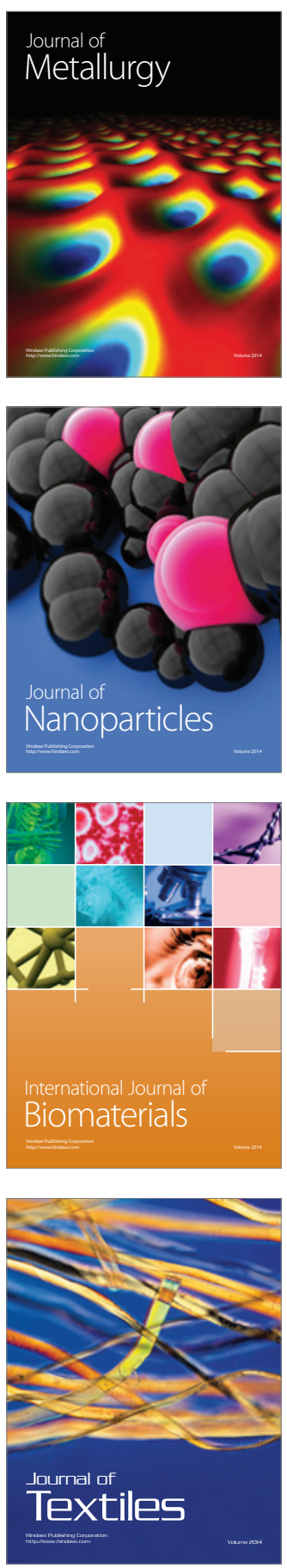